\title{
Transmission of SARS-CoV-2 Involving Residents Receiving Dialysis in a Nursing Home - Maryland, April 2020
}

\author{
Benjamin F. Bigelow ${ }^{1, *}$; Olive Tang, PhD ${ }^{1, *}$; Gregory R. Toci ${ }^{1}$; Norberth Stracker, MS ${ }^{1,2}$; Fatima Sheikh, MD ${ }^{1}$; Kara M. Jacobs Slifka, MD ${ }^{3}$; \\ Shannon A. Novosad, $\mathrm{MD}^{3}$; John A. Jernigan, $\mathrm{MD}^{3}$; Sujan C. Reddy, MD³ ${ }^{3}$ Morgan J. Katz, $\mathrm{MD}^{1}$
}

\section{On August 11, 2020, this report was posted as an MMWR Early Release on the MMWR website (https://www.cdc.gov/mmwr).}

SARS-CoV-2, the virus that causes coronavirus disease 2019 (COVID-19), can spread rapidly in nursing homes once it is introduced $(1,2)$. To prevent outbreaks, more data are needed to identify sources of introduction and means of transmission within nursing homes. Nursing home residents who receive hemodialysis (dialysis) might be at higher risk for SARS-CoV-2 infections because of their frequent exposures outside the nursing home to both community dialysis patients and staff members at dialysis centers (3). Investigation of a COVID-19 outbreak in a Maryland nursing home (facility A) identified a higher prevalence of infection among residents undergoing dialysis $(47 \%$; 15 of 32$)$ than among those not receiving dialysis $(16 \% ; 22$ of 138$)(\mathrm{p}<0.001)$. Among residents with COVID-19, the 30-day hospitalization rate among those receiving dialysis (53\%) was higher than that among residents not receiving dialysis $(18 \%)(\mathrm{p}=0.03)$; the proportion of dialysis patients who died was $40 \%$ compared with those who did not receive dialysis $(27 \%)(\mathrm{p}=0.42)$. Careful consideration of infection control practices throughout the dialysis process (e.g., transportation, time spent in waiting areas, spacing of machines, and cohorting), clear communication between nursing homes and dialysis centers, and coordination of testing practices between these sites are critical to preventing COVID-19 outbreaks in this medically vulnerable population.

In April 2020, a COVID-19 outbreak occurred at a Maryland nursing home (facility A), a 200-bed skilled nursing facility specializing in postacute and long-term care, with an independently operated dialysis center co-located on site. In Maryland, during the month of April, approximately $25 \%$ of all SARS-CoV-2 tests had positive results when considering the rolling 7-day average, and approximately half of nursing homes in the state had active outbreaks. ${ }^{\dagger}$ The Maryland Department of Health conducted SARS-CoV-2 testing for symptomatic nursing home residents with a 3-5-day turnaround time for results. Because of the evolving outbreak and limited testing capacity at the health department, a Johns Hopkins response team provided SARS-CoV-2 testing with a 24-hour turnaround

\footnotetext{
${ }^{*}$ These authors contributed equally to this work.

${ }^{\dagger}$ https://coronavirus.maryland.gov/.
}

time for all facility A residents who had not previously had a positive test result within the past 48 hours. On April 30, SARS-CoV-2 testing was conducted among all facility A residents, and the prevalences among patients receiving and those not receiving dialysis and by floor of residence in facility A were assessed. All statistical analyses were performed using chisquare tests $(\mathrm{p}<0.05)$ with Stata statistical software (version 16; StataCorp, LLC).

\section{Investigation and Findings}

On April 16, 2020, the facility census was 170; 75\% of residents resided in double-occupancy rooms. Thirty-two (19\%) residents were receiving dialysis at the co-located dialysis center. The two schedules for dialysis were Monday, Wednesday, and Friday or Tuesday, Thursday, and Saturday, with three 4-hour shifts per schedule. Shifts overlapped appointment times and residents remained in a dialysis waiting room until their appointment. Facility A residents accounted for $40 \%$ of dialysis patients at the center; other patients were from the surrounding community and were scheduled simultaneously with facility A residents.

By April 1, per an order by the Maryland Governor, facility A and the dialysis center required universal surgical masks for all staff members, cancelled group activities and group dining, and prohibited visitors. Staff members were screened for symptoms (e.g., shortness of breath, cough, fever, myalgias, headache, diarrhea, and loss of taste or smell) and their temperature was measured before each shift and being permitted to work. Residents of the nursing home were screened every 8 hours; community dialysis patients were screened before their dialysis appointment.

On April 16, a resident at facility A developed an elevated temperature and malaise and subsequently had a positive result for SARS-CoV-2 RNA by reverse transcription-polymerase chain reaction (RT-PCR) testing of a nasopharyngeal swab specimen. This resident (the index patient) received dialysis on the Tuesday, Thursday, and Saturday schedule during shift 2. The patient received dialysis on April 18, and after receiving a positive test result, was transferred to a designated COVID-19 area in another nursing home (Figure 1).

During the following week (beginning April 20), the Maryland Department of Health tested 47 symptomatic 
residents of the nursing home and 10 symptomatic staff members (symptoms were defined as any of the following: fever $>99^{\circ} \mathrm{F}\left[37.2^{\circ} \mathrm{C}\right]$, cough, malaise, headache, or upper respiratory symptoms); 11 residents and three staff members had positive test results for SARS-CoV-2. Two of the infected residents received dialysis on the Monday, Wednesday, and Friday schedule, one each on shifts 2 and 3. All staff members with positive test results were excluded from work.

On April 21, the dialysis center attempted to cohort all residents into four groups: 1) patients with confirmed COVID-19; 2) symptomatic persons with SARS-CoV-2 test results pending; 3) potentially exposed but asymptomatic persons; and 4) asymptomatic, nonexposed persons. Per cohorting strategy, when possible, these groups received dialysis during different shifts; however, because of scheduling constraints, groups 1 and 2 could receive dialysis on the same shift, as could groups 3 and 4 . Universal masking was strongly recommended for patients in the dialysis center; however, the center reported patients often had difficulty wearing masks for the entire session. Dialysis center staff members caring for patients with COVID-19 were required to wear gowns, masks, gloves, and eye protection. Efforts were made to separate dialysis machines by 6 feet ( 2 meters), but because of space limitations, this was not always possible.

On April 30, among the facility's 164 residents, 152 (93\%) had nasopharyngeal specimens tested for SARS-CoV-2 with RT-PCR; three residents refused testing, and nine had previously received positive SARS-CoV-2 test results. Symptom status at the time of universal testing was recorded based on discussion with facility staff members. Among the 152 residents who received testing, 25 (16\%) additional SARS-CoV-2 infections were identified, including in $12(41 \%)$ of the 29 remaining residents who were receiving dialysis and in $13(11 \%)$ of the 123 remaining residents who were not receiving dialysis. Among the 25 newly identified cases, $18(72 \%)$ persons were asymptomatic at the time of testing, including seven of 12 and 11 of 13 residents who did and did not receive dialysis, respectively. Two dialysis technicians subsequently became symptomatic, received positive test results (May 1 and May 4) and self-isolated at home. Overall, 40 COVID-19 cases were identified in facility $A$ in 37 residents and three staff members.

As of April 30, 15 of 32 (47\%) residents receiving dialysis had positive test results, compared with 22 of 138 (16\%) who did not receive dialysis ( $\mathrm{p}<0.001$, chi-squared test) (Table). The prevalence of SARS-CoV-2 infection among residents on the second floor of facility A (33 of $81 ; 41 \%$ ) was significantly higher than that among residents on the first floor (four of 89; $4.5 \%)(\mathrm{p}=<0.001)($ Figure 2).
Among residents with SARS-CoV-2 infection, those receiving dialysis were more often hospitalized within 30 days of receiving a positive test result (eight of 15) compared with those not receiving dialysis (four of $22 ; 18 \%)(\mathrm{p}=0.03$ ). Among residents with SARS-CoV-2 infection, six of 15 residents receiving dialysis and six of $22(27 \%)$ residents not receiving dialysis died within 30 days of diagnosis $(\mathrm{p}=0.42)$. Information on cause of death or comorbidities was not available for residents who died.

\section{Public Health Interventions}

Facility A closed to new admissions after the first case was identified on April 16 and did not accept new admissions until May 8. Testing for symptomatic residents and staff members was conducted during April 16 -29. Follow-up facility-wide testing for all residents who had not previously had test results positive for SARS-CoV-2, regardless of symptoms, was conducted on April 30; however, because of testing limitations, asymptomatic staff members and community dialysis patients were not tested. To mitigate transmission among residents, following guidance from the local health department, the facility cohorted residents by test results. All residents, regardless of COVID-19 status, were isolated in their rooms while the facility remained in active outbreak status. Staff members were required to wear personal protective equipment for care of all residents with positive test results and those under observation. Residents with SARS-CoV-2 infection receiving dialysis were scheduled separately from residents who had negative test results.

\section{Discussion}

During a COVID-19 outbreak investigation at a skilled nursing facility in Maryland, testing identified infections in both residents who were and were not receiving dialysis, but disease prevalence was significantly higher among residents receiving dialysis and among residents on the second floor compared with those not receiving dialysis and those on the first floor.

Residents leaving their rooms for dialysis could be a potential source of SARS-CoV-2 introduction into the nursing home and might pose an underrecognized source of transmission, both in the dialysis center and in the nursing home. Better monitoring and understanding of the risks associated with residents who regularly leave the facility for outpatient health care is needed. Implementing procedures that ensure use of masks, social distancing, and improved ventilation during transportation and in waiting areas is important for preventing SARS-CoV-2 transmission.

Nursing home residents who undergo dialysis are a particularly vulnerable population $(3,4)$. Compared with other residents, they often have more underlying medical 


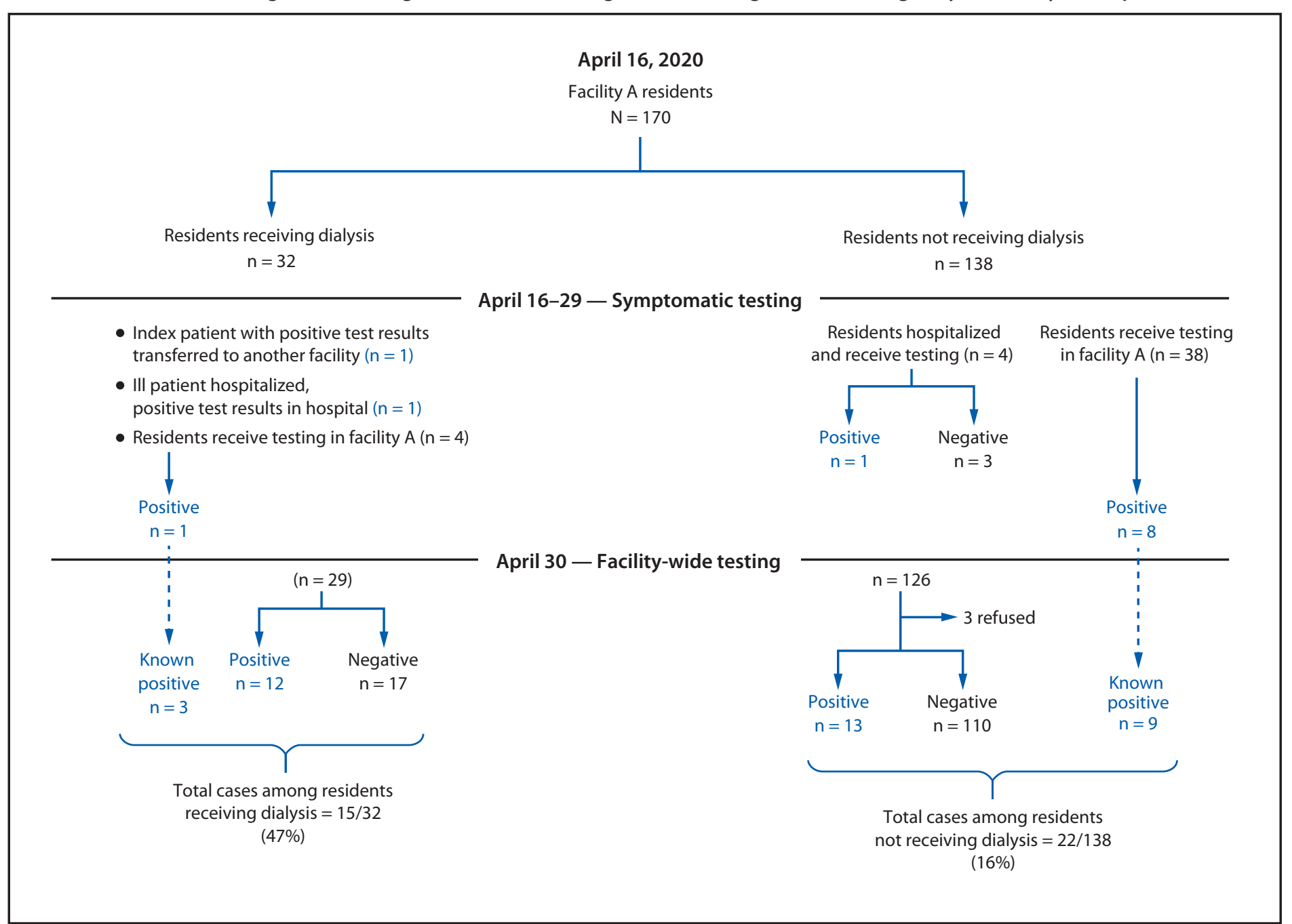

conditions, many of which have been associated with more severe SARS-CoV-2 infections, including diabetes mellitus, hypertension, and heart disease $(5,6)$. This population might also be more frequently exposed to persons outside the nursing home, including community dialysis patients and dialysis center staff members.

Identifying the definitive source for this outbreak or tracing the chain of subsequent transmission was not possible. For example, many residents receiving dialysis were housed on the second floor of the nursing home, and transmission might have occurred within the nursing home, at the dialysis center, or during transportation between the two locations (e.g., in the closed confines of an elevator). Given that shifts overlapped appointment times at the dialysis center, before their dialysis appointments residents might spend time in a waiting area where additional exposures might occur. Further, whereas the first identified cases occurred among residents who were
TABLE. Number of residents who had positive test results for SARS-CoV-2 RNA among facility A residents $(\mathrm{N}=170)$, overall and by residence floor and dialysis schedule - Maryland, April 16-30, 2020

\begin{tabular}{lcr}
\hline Characteristic & No. of residents & No. (\%) of cases \\
\hline Dialysis status, all residents & 138 & \\
Not receiving dialysis & 32 & $22(16)$ \\
Receiving dialysis & $15(47)$ \\
Facility residence (residents receiving dialysis only) & \\
First floor & 7 & $2(29)$ \\
Second floor & 25 & $13(52)$ \\
Dialysis schedule & & \\
Monday/Wednesday/Friday & 19 & $9(47)$ \\
Shift 1 & 4 & $0(0)$ \\
Shift 2 & 3 & $1(33)$ \\
Shift 3 & 12 & $8(67)$ \\
Tuesday/Thursday/Saturday & 13 & $6(46)$ \\
Shift 1 & 6 & $3(50)$ \\
Shift 2 & 6 & $2(33)$ \\
Shift 3 & 1 & $1(100)$ \\
\hline
\end{tabular}






Abbreviations: COVID-19 = coronavirus disease 2019; $D=$ room of resident receiving dialysis.

* All dialysis treatments were completed in the dialysis center, which was co-located on site. Symptom-based testing referred to targeted testing of residents who were experiencing at least one of the following symptoms: fever $>99^{\circ} \mathrm{F}\left(37.2^{\circ} \mathrm{C}\right)$, cough, malaise, headache, or upper respiratory symptoms. Facility-wide testing refers to the testing of all facility A residents who had not previously had test results positive for SARS-CoV-2, regardless of symptoms. 
receiving dialysis, given the COVID-19 incubation period of up to 14 days and delayed testing among other residents and staff members, the definitive source of introduction remains unclear. The prevalence of asymptomatic infections poses additional challenges to identifying the source of introduction and tracing transmission through the facility $(7,8)$.

The findings in this report are subject to at least three limitations. First, no observations of infection control and prevention practices were conducted in the dialysis center, limiting the ability to identify breaches that might have contributed to transmission. Second, the impact of residents leaving the facility for other medical appointments was not assessed. Finally, because of limited testing capacity, testing for all asymptomatic staff members in the nursing home was not performed, and records of activities for infected staff members were not available.

Effective and continual communication between dialysis centers and nursing homes is important to preventing SARS-CoV-2 transmission. If nursing homes rapidly notify dialysis centers of residents who have positive test results and those with suspected infection, dialysis centers can cohort residents (e.g., inform recommended use of personal protective equipment and provide dialysis for residents with positive test results during last shift of day with terminal cleaning) and limit exposure to others in the dialysis center (9). Likewise, if dialysis centers notify the nursing home in a timely manner of any community dialysis patients or dialysis staff members who had positive test results, nursing homes can perform facility-wide testing to detect asymptomatic cases and take recommended precautions (e.g., placing all exposed patients in quarantine) (9). Dialysis centers and nursing homes might benefit from closely reviewing the entire dialysis process, from residents leaving the facility to discharging them after dialysis, to identify practices that could contribute to SARS-CoV-2 transmission. Nursing homes might consider placing residents who undergo dialysis in single rooms close to the dialysis center with increased monitoring given their higher risk for infection. Dialysis centers and nursing homes are closely connected with a shared patient population; therefore, early identification of cases coupled with aggressive infection prevention and control actions are needed to protect medically vulnerable populations in both locations.

\section{Acknowledgments}

Johns Hopkins ambulatory response team, facility staff members, and residents.

Corresponding author: Benjamin F. Bigelow, benbigelow@jhmi.edu.

\section{Summary}

What is already known about this topic?

Residents of long-term care facilities have high COVID-19associated morbidity and mortality. More information is needed about SARS-CoV-2 introduction and transmission in nursing homes.

What is added by this report?

Investigation of a COVID-19 outbreak in a Maryland nursing home identified a significantly higher prevalence among residents receiving dialysis (47\%) than among those not receiving dialysis (16\%); $72 \%$ were asymptomatic at the time of testing.

What are the implications for public health practice?

Nursing home residents undergoing dialysis might be at a higher risk for SARS-CoV-2 infection because of exposures to staff members and community dialysis patients. Attention to infection control practices and surveillance in nursing homes and dialysis centers is critical to preventing nursing home COVID-19 outbreaks.

${ }^{1}$ Department of Medicine, Johns Hopkins University School of Medicine, Baltimore, Maryland; ${ }^{2}$ Division of Population Health and Disease Prevention, Baltimore City Health Department, Baltimore, Maryland; ${ }^{3}$ CDC COVID-19 Response Team.

All authors have completed and submitted the International Committee of Medical Journal Editors form for disclosure of potential conflicts of interest. No potential conflicts of interest were disclosed.

\section{References}

1. Arons MM, Hatfield KM, Reddy SC, et al.; Public Health-Seattle and King County; CDC COVID-19 Investigation Team. Presymptomatic SARS-CoV-2 infections and transmission in a skilled nursing facility. N Engl J Med 2020;382:2081-90. https://doi.org/10.1056/NEJMoa2008457

2. Sanchez GV, Biedron C, Fink LR, et al. Initial and repeated point prevalence surveys to inform SARS-CoV-2 infection prevention in 26 skilled nursing facilities_-Detroit, Michigan, March-May 2020. MMWR Morb Mortal Wkly Rep 2020;69:882-6. https://doi.org/10.15585/ mmwr.mm6927e1

3. Corbett RW, Blakey S, Nitsch D, et al.; West London Renal and Transplant Centre. Epidemiology of COVID-19 in an urban dialysis center. J Am Soc Nephrol 2020;31:1815-23. https://doi.org/10.1681/ASN.2020040534

4. Rombolà G, Heidempergher M, Pedrini L, et al. Practical indications for the prevention and management of SARS-CoV-2 in ambulatory dialysis patients: lessons from the first phase of the epidemics in Lombardy. J Nephrol 2020;33:193-6. https://doi.org/10.1007/s40620-020-00727-y

5. Vanholder R, Massy Z, Argiles A, Spasovski G, Verbeke F, Lameire N; European Uremic Toxin Work Group. Chronic kidney disease as cause of cardiovascular morbidity and mortality. Nephrol Dial Transplant 2005;20:1048-56. https://doi.org/10.1093/ndt/gfh813

6. CDC. Coronavirus disease 2019 (COVID-19): evidence used to update the list of underlying medical conditions that increase a person's risk of severe illness from COVID-19. Atlanta, GA: US Department of Health and Human Services, CDC; 2020. https://www.cdc.gov/coronavirus/2019ncov/need-extra-precautions/evidence-table.html 
7. Gandhi M, Yokoe DS, Havlir DV. Asymptomatic transmission, the Achilles' heel of current strategies to control Covid-19. N Engl J Med 2020;382:2158-60. https://doi.org/10.1056/NEJMe2009758

8. Bigelow BF, Tang O, Barshick B, et al. Outcomes of universal COVID-19 testing following detection of incident cases in 11 long-term care facilities. JAMA Intern Med 2020. https://doi.org/10.1001/jamainternmed.2020.3738
9. CDC. Coronavirus disease 2019 (COVID-19): interim additional guidance for infection prevention and control recommendations for patients with suspected or confirmed COVID-19 in outpatient hemodialysis facilities. Atlanta, GA: US Department of Health and Human Services, CDC; 2020. https://www.cdc.gov/coronavirus/2019ncov/hcp/dialysis.html 